\title{
A chaotically driven model climate: extreme events and snapshot attractors
}

\author{
T. Bódai ${ }^{1}$, Gy. Károlyi ${ }^{2}$, and T. Tél ${ }^{1}$ \\ ${ }^{1}$ Institute for Theoretical Physics, Eötvös University, Pázmány P. s. 1/A, Budapest, 1117, Hungary \\ ${ }^{2}$ Dept. of Structural Mechanics, Budapest Univ. of Technology and Economics, Múegyetem rkp. 3., Budapest, 1111, Hungary
}

Received: 20 April 2011 - Revised: 14 July 2011 - Accepted: 15 August 2011 - Published: 7 September 2011

\begin{abstract}
In a low-order chaotic global atmospheric circulation model the effects of deterministic chaotic driving are investigated. As a result of driving, peak-over-threshold type extreme events, e.g. cyclonic activity in the model, become more extreme, with increased frequency of recurrence. When the characteristic time of the driving is comparable to that of the undriven system, a resonance effect with amplified variance shows up. For very fast driving we find a reduced enhancement of variance, which is also the case with white noise driving. Snapshot attractors and their natural measures are determined as a function of time, and a resonance effects is also identified. The extreme value statistics of group maxima is found to follow a Weibull distribution.
\end{abstract}

\section{Introduction}

In the modeling of climate systems the approach of stochastic parametrization is a disputed topic and active area of research (Wilks, 2008). Any model of such a complex system is inevitably a poor approximation of reality since many parameters, or perhaps even underlying physical processes, are not precisely known. Therefore it is worth simulating these uncertainties by the inclusion of proper noise terms into the parameters of the dynamics, as suggested by Ghil et al. (2008) and Chekroun et al. (2010). In their studies stochasticity is represented by white noise. Our aim is to extend the stochastic approach from using white noise to using temporally chaotic drivings. The novelty of this is that, although the driving remains random-like, its time scale need not to be much shorter than that of the basic dynamics, but it can be comparable or even longer than that.

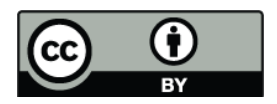

Correspondence to: T. Bódai (bodai@general.elte.hu)
For a first test of the effects of replacing white noise by chaotic driving a conceptual model of the atmosphere is used here, Lorenz's global atmospheric circulation model (L84) (Lorenz, 1984). This low-order model is not only appealing (Shil'nikov et al., 1995; Provenzale and Balmford, 1999; Tél and Gruiz, 2006, p. 293; Freire et al., 2008), but it can be derived from the quasi-geostrophic equations governing the large-scale motion of the atmosphere (Roebber, 1995). As a driving, we choose a paradigmatic chaotic signal, the first component of the classical Lorenz equations (L63) (Lorenz, 1963) coupled in an additive manner. The coupling of low-order models has already been used in the climate context, see e.g. the works of Palmer (1993) and Ghil and Jiang (1998). We focus on changes in the variability of the system, relative to either the undriven or the noisy case.

The study of extreme events is timely, and has already been investigated in elementary chaotic models (Nicolis et al., 2006; Nicolis and Nicolis, 2009). In our chaotically driven model climate we study extreme events, defined in a peak-over-threshold sense (Blender et al., 2008), and their return time statistics as a function of the time scales and the threshold level. We demonstrate that with chaotic driving, relative to the undriven system, (i) seldom occurring events become more extreme, and (ii) the return times of moderately extreme events might become shorter or longer depending on the time scale of driving and the threshold level - while they are always shorter with white noise. Given that the atmospheric circulation model L84 derives from fluid dynamics (Roebber, 1995), our results prompt the importance of using a proper - perhaps chaotic - parametrization for more realistic predictions.

The concept of pullback attractors of driven continuoustime dynamical systems (Arnold, 1998; Ghil et al., 2008) has been suggested (Chekroun et al., 2010) as a promising tool to describe the variance of white noise-driven climate dynamics in a novel way. They propose that one should investigate

Published by Copernicus Publications on behalf of the European Geosciences Union and the American Geophysical Union. 
an ensemble of trajectories (a broadly applied method in the spirit of ensemble forecasting), all subject to the same realization of noise. After some time, the ensemble traces out a pullback attractor, and also determines a distribution on it. The attractor is changing continuously in time, and typically remains chaotic throughout its evolution. The pullback attractors, that are the fractal building blocks of fuzzy noise-induced chaotic attractors (Bódai et al., 2011), provide a more detailed description of the process than the traditional methods.

In the dynamical systems community, a practically equivalent concept, that of the snapshot attractors, has been known for many years (Romeiras et al., 1990). The idea is particularly well suited for understanding the advection of passive particles in random flows (Sommerer and Ott, 1993; Jacobs et al., 1997; Neufeld and Tél, 1998), and explains experimental findings (Sommerer and Ott, 1993). In this setting the driving might also be some chaotic process.

In general, a snapshot or pullback attractor is an object which attracts any trajectories initialized in the infinitely remote past within a basin of attraction (Ghil et al., 2008). A snapshot attractor can be either a simple object or a fractal.

In the following, extreme-event-related return time statistics is pursued also in the snapshot attractor framework. We find that the magnitude of seldom occurring events also depends on the time scale of chaotic driving in a non-monotonic manner, revealing a resonance effect. In terms of snapshot probability distributions this resonance manifests itself in sharp peaks.

The paper is organized as follows. Next, in Sect. 2, the model is introduced and explained. In Sect. 3 the concept of snapshot attractors is reviewed in more details, and the technique of their construction is described. Measures of extremal behavior in the snapshot framework are then identified. Subsequently, in Sect. 4, the results of extreme event return time statistics are presented, in the snapshot ensemble as well as the single trajectory framework. The extreme value statistics is identified as Weibullian. In the end, in Sect. 5, we discuss our results and draw conclusions.

\section{Model}

The model to be studied reads as follows:

$$
\left.\begin{array}{l}
\dot{x}=-y^{2}-z^{2}-a x+a\left(F_{0}+A \tilde{x}\right), \\
\dot{y}=x y-b x z-y+1, \\
\dot{z}=x z+b x y-z,
\end{array}\right\}
$$

The physical content of Eq. (L84) is that the time-dependent solar forcing

$$
F(t)=F_{0}+A \tilde{x}(t)
$$

creates a temperature difference between the equator and the pole, which influences most directly the wind speed of the Westerlies represented by $x$. As a counter effect to thermal imbalances, cyclonic activity facilitates poleward heat transport, two modes of which are represented by $y$ and $z$. By definition, the variables correspond to global averages, and there is no possibility for studying local quantities as in more realistic models or by measurement data.

Note that the coupling between L84 an L63 acts one way only, that is, L84 does not influence L63. The coupled system of L84 and L63 is autonomous. Being interested in the climate model L84, we can consider it as a non-autonomous, or driven, subsystem subject to a time-dependent solar radiation $F(t)$ (1) where the temporally irregular part of the driving follows from the $\tilde{x}$-component of L63 for $A \neq 0$.

For the parameter setting we take the common choice: $a=1 / 4, b=4$ (L84) and $\sigma=10, \rho=28, \beta=8 / 3$ (L63). Parameter $a$ is the ratio of the damping times of the Westerlies and the cyclonic eddies, while $b$ is the ratio of relaxation times of respectively the displacement and amplification of eddies by the Westerlies. Both sets of equations appear in a dimensionless form with the time unit in L84 corresponding to about 5 days, the characteristic damping time of eddies. The time-scale factor $\tau$ is viewed as the ratio of the characteristic times in L63 and L84. The choice of $\tau \approx 5$ can be interpreted as a daily influence of, say, convectional origin, while $\tau \approx 1 / 73$ corresponds with an annual time scale of driving. The coupling strength $A$ is set to realize rather strong perturbation, superimposed onto a static part of the forcing, $F_{0}$. The standard choices with $A=0$ are (Lorenz, 1984): $F_{0}=6$ for summer, and $F_{0}=8$ for winter. For the driven case we choose $F_{0}=8$ and $A=0.025$. Given that $\tilde{x}(t) \in[-17.5,17.5]$, we have $F(t) \in[7.56,8.44]$. In Eq. (1) $A \tilde{x}$ may be interpreted as the variation of the albedo either through cloud formation due to varying weather conditions $(\tau \geq 1)$, or orbital eccentricity $(\tau \ll 1)$. More generally $F(t)$ can also be related to the strength of the green house effect.

\section{Snapshot attractors}

The autonomous L84 system $(A=0)$ exhibits regular summer and chaotic winter (see bifurcation diagram in Fig. 1e). The dynamics can be conveniently visualized by a 2-D Poincaré section of the attractor, taken traditionally as the $z=0$ section.

In the driven, nonautonomous system, when using a single trajectory, the attractor on the $z=0$ Poincare section (PS) is fuzzy. In contrast, if an ensemble of trajectories of the L84 system is followed - with a smooth initial distribution 
(a)

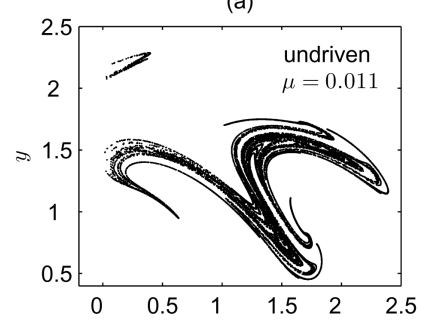

(c)
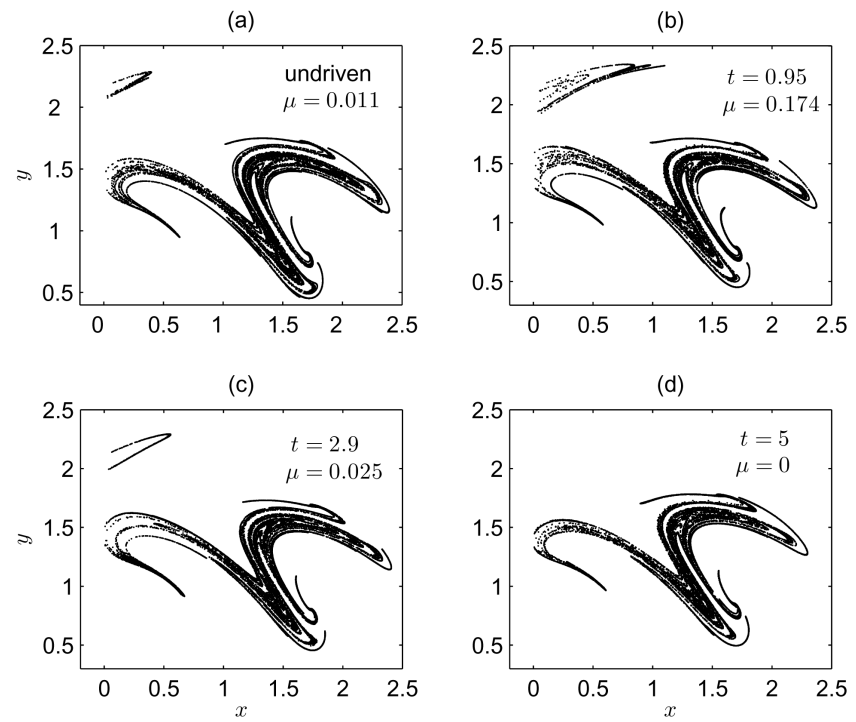

(d)

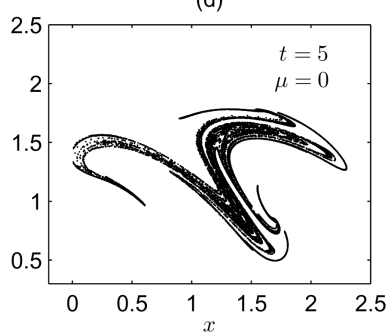

(e)

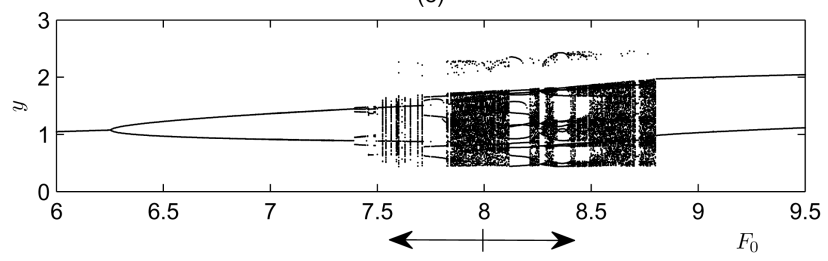

Fig. 1. (a) Chaotic attractor on the Poincare section $z=0, \dot{z}>0$, of the undriven system $(A=0)$ with $F_{0}=8$ and $(\mathbf{b}-\mathbf{d})$ snapshot attractors of the chaotically driven system $(A=0.025)$ with $\tau=1$, taken at the indicated times (when $F$ takes values of 7.89, 8.16, and 7.65 , respectively, and the instantaneous measure $\mu$ of the island is also indicated). (e) Bifurcation diagram of the $y$ coordinate with $A=0$. The arrow indicates the range swept by the signal $F$ given by Eq. (1). Dots around $y \approx 2$ correspond to a chaotic "island" or points from periodic attractors.

at $t_{0}=0-$ up to a certain time $t \gg 1$, the momentary coordinates $x, y, z$ trace out a fractal object, the snapshot attractor, whose shape is continuously changing over time $t$. Its $z=0$ section is also a fractal, which is commonly referred to also as a snapshot attractor. The ensemble is necessarily of finite size in numerical studies, and particles fall on the surface of intersection with zero probability at any given time. This situation can be catered for naively by considering a $\delta z$ neighborhood of the chosen sectioning surface, viewing particles from within a "thick slice". The problem with this technique is, however, that the resulting distribution of retained particles does not represent the distribution over the true instantaneous PS. An appropriate technique to overcome this is the following. The unique time instants when the particles, each and every one, cross the surface are recorded, and those particles together are retained to form a PS whose crossing time fall in a particular time interval of length $\delta t \ll 1$. We use $\delta t=0.01$.
Snapshot attractors are shown in Fig. 1b-d in various time instants. It is remarkable that the snapshot attractors of this model remain in the first quadrant of the $x-y(z=0)$ plane for any time. The time is measured from after 100 time units when the $N=10^{7}$ particles, initially evenly distributed in $x \in[1.15,1.25], y \in[1,1.1], z=0$, already reached the 3-D snapshot attractor. (A typical convergence time to the attractor is estimated to be 30 time units.) The equations were simulated using a fourth order Runge-Kutta integrator, with a fixed time step of $h=0.005$. Note that the pattern changes with time, but keeps the fractal character. The snapshot attractor consists of a "mainland", slightly displaced and distorted over time, and occasionally an "island" which can grow large or disappear for finite periods of time. Thus, the island is identified as a feature in which the deviation of the driven system from the undriven one (Fig. 1a) is clearly manifested, since the PS and hence the island certainly never changes in the undriven case.

One might ask if the appearance of the island is not an artefact due to a special choice of the PS. To answer this question a 3-D snapshot attractor is shown in Fig. 2, whose PS is shown in Fig. 1c. It can be seen that its "front view" is not centrally symmetric. The maximal extension of the attractor consist in a bulge, which can be regarded to represent extremal behavior. When traditionally taking an intersection with $z=0$ for the better visualization of the fractality, it is this bulge that appears as an island. It is fortunate that the traditional section $z=0$ properly reflects the presence of the bulge.

In physical terms any region belonging to only large values of $y$ or $z$ represents extreme cyclonic activity in the model. Since the bulge or the island is associated with large $y(>1.9)$ values, and because of their unique shape in this model, the island is particularly well suited to represent extreme events in this model. The natural measure $\mu$ of the island is the probability that a trajectory of the ensemble visits the island on the PS. Numerically it is well approximated by the relative number of particles hitting the region of the island on the PS at a given instant of time. In Fig. 3 time series of $\mu$ is shown for various values of $\tau$. These are segments of longer time series of length $t_{f}=500$. In the diagrams, the upper horizontal line indicates the maximal island measure of all those undriven systems $(A=0)$ for which $F_{0}$ is from the range of the arrow of Fig. 1e. Regular regimes where no islands exist are excluded. It is perhaps the most remarkable feature of the diagram that the maximal value 0.044 of the island measure with constant $F=F_{0}$ (found at $F_{0}=8.33$ ) is exceeded regularly and significantly. The island measure $\mu$ can range up to 0.6 or more with $\tau=1$ (not shown). The snapshot in Fig. 1b represents a case with $\mu=0.174$. We note that defining an island by $y>1.9$ results in a maximal island "measure" of $1 / 13=0.077$ for the dominant period-13 attractor in the periodic window. This value is also exceeded by far. 

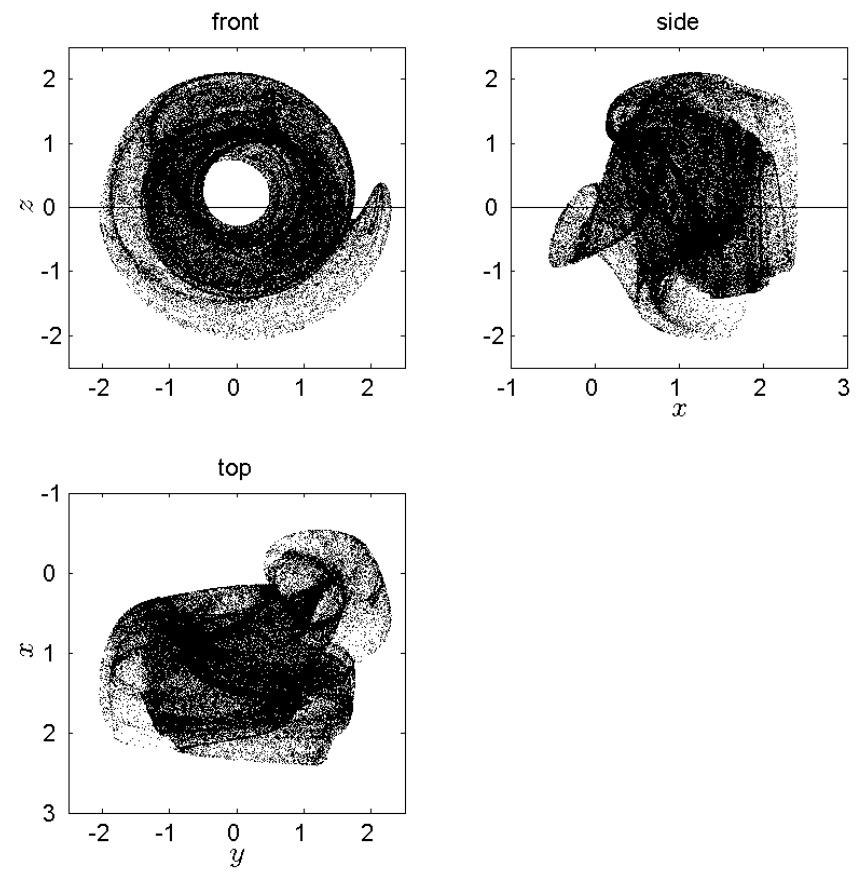

Fig. 2. Front, side and top views of the $3-\mathrm{D}$ snapshot attractor at $t=$ 2.9, the $z=0$ section of which is shown in Fig. 1c. The horizontal line indicates the $z=0$ surface of intersection.

For comparison we also studied L84 driven by white noise: $\tilde{x}$ in L84 is replaced by a homogeneously distributed random variable in $[-17.5,17.5]$. It is worth noting that in this case the island on the PS was found to never disappear. This in itself is a hint of the fact that the variance of extremes is larger with chaotic driving.

By a mere observation of the islands it is surprising that their measure can be comparable with that of the full attractor. The distribution on the snapshot attractor, however, explains the phenomenon (Fig. 4b), showing a strong accumulation of particles in some parts of the island. For comparison, Fig. 4a shows the undriven case from Fig. 1a with $\mu=0.011$.

The probability distribution $P_{y}$ of $y$ for the 3-D snapshot attractors, whose PS's are displayed in Fig. 1b-d, is also evaluated (Fig. 5). With chaotic driving there is a clear tendency of the distribution to have sharp peaks, which are displaced and resized over time. These are similar features to those exposed in Fig. 4.

It is worth emphasizing that for the range of parameters investigated the snapshot attractor is never found to be regular in spite of the fact that the admittable $F$ values cover several periodic windows of the undriven problem (see Fig. 1e); i.e. chaos suppresses the regular behavior.
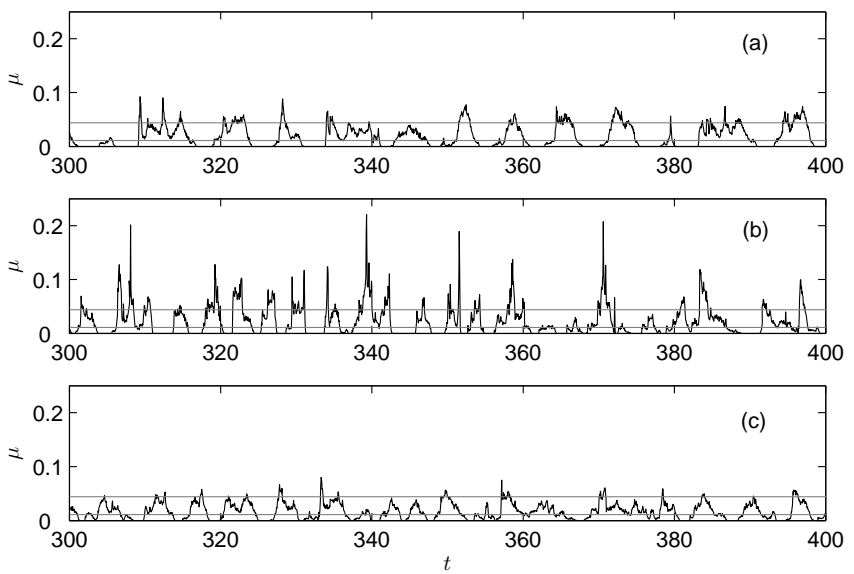

Fig. 3. Time series of the island measure $\mu$ for (a) $\tau=1 / 10$, (b) $\tau=$ 1 , and (c) $\tau=10$. The measure of the island is plotted with a time increment of 0.05 . The number of points in the sections is varying between about 5000 and 16000 (with $N=10^{6}$ ). Upper horizontal line indicates the maximal value of $\mu=0.044$ for the undriven system with fixed $F_{0}$ in $[7.56,8.44]$, i.e. in the interval in which the driving changes; the lower one indicates $\mu\left(F_{0}=8\right)=0.011$.
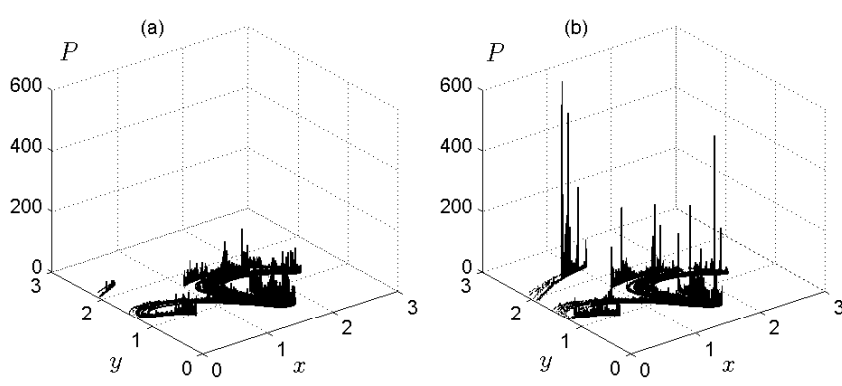

Fig. 4. The natural distribution $P$ of the attractor on the PS indicates the strong differences in the island measure. The distributions in panels (a) and (b) correspond to Fig. 1a and b with $\mu=0.011$ and $\mu=0.174$, respectively. A number of 250 bins are used in both $x$ and $y$ directions, and the PS's involve about 35000 attractor points each.

\section{Extreme event recurrence time and extreme value statistics}

\subsection{Recurrence time statistics}

\section{Ensemble (snapshot) framework}

The statistics of extreme events in terms of the time series of $\mu(t)$ can be studied in a peak-over-threshold sense with some threshold level $\mu_{T}$. The return time $t_{r}$ of an extreme event is the time interval between a pair of subsequent descending and ascending branches of the time series belonging to a chosen value of $\mu_{T}$. The dependence of mean return times $\bar{t}_{\mathrm{r}}$ on threshold $\mu_{T}$ is displayed in Fig. 6. Each curve is shown up to a threshold value $\mu_{\max }$ beyond which the statistics is 
(a)

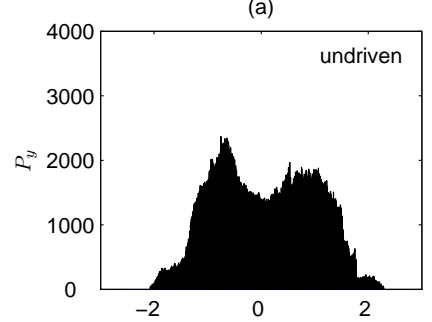

(c)

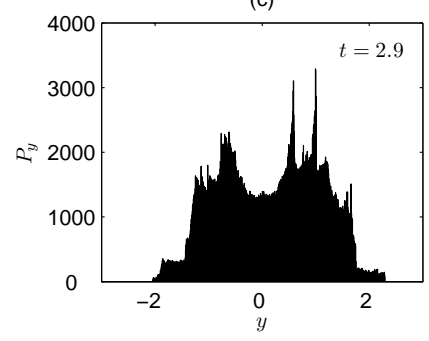

(b)

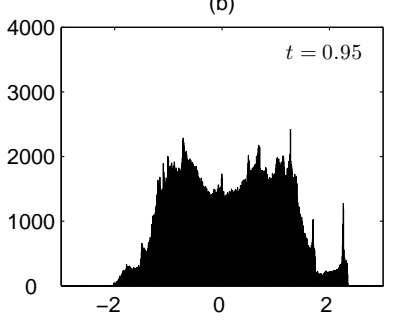

(d)

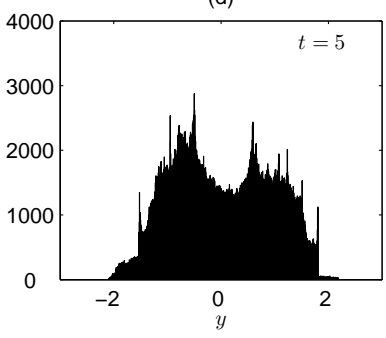

Fig. 5. Probability distribution (histogram) $P y$ of $y$ on the 3-D snapshot attractors corresponding to Fig. 1. A bin size of 0.005 is used to construct the histograms.

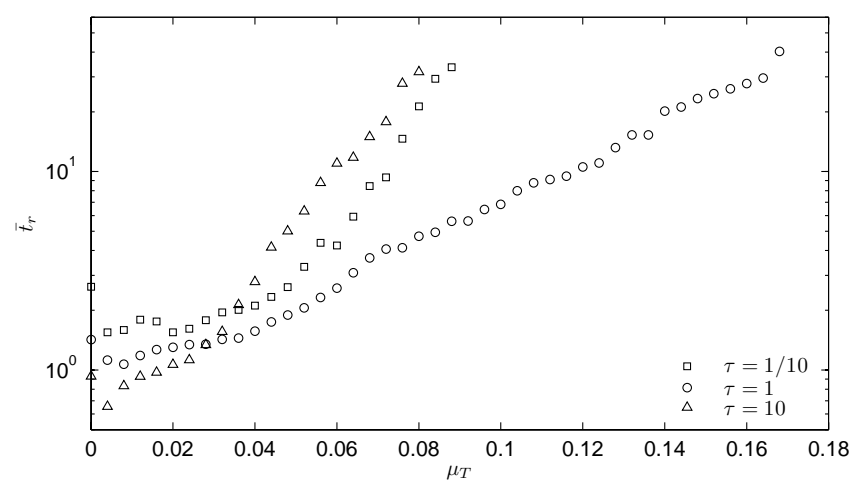

Fig. 6. Dependence of the mean return times $\bar{t}_{\mathrm{r}}$ on the threshold island measure $\mu_{T}$ of the snapshot attractors, based on a time series of length $t_{f}=500$ time units (parts of them shown in Fig. 3).

poor because of the finite length $t_{\mathrm{f}}=500$. For all $\tau$ 's studied, a close to exponential scaling can be observed beyond a certain threshold level. The $\tau$-dependence is nonmonotonic, which can be seen as a resonance effect; the return times are the shortest in case of $\tau=1$, when extreme events tend also to be much more extreme. Indeed, more high peaks can be seen in Fig. $3 b$ than in a and c. Near zero level $(\mu<0.03)$ events, on the other hand, reveal monotonic dependence of $\bar{t}_{\mathrm{r}}$ on $\tau$, also consistent with the time series. A minimum occurs at these low levels, presumably because of the one-sided feature of the time series: lower turning points are missing due to the disappearances of the island.

\section{Single trajectory framework}

The effects of chaotic driving and its time scale $\tau$ are studied also in terms of the continuous time-dependence of $y$. Qualitatively the time series (not shown) look very similar for the different $\tau$ 's. However, the dependence of the mean return times on a threshold $y_{\mathrm{T}}$, displayed in Fig. 7, admits more difference. Each curve is shown up to a maximal threshold value $y_{\max }$ beyond which the statistics is poor. Two main features can be seen: (i) The driving turns out to make the dynamics more prone to extremes, both in terms of increased levels of $y_{\max }$, i.e. amplified variances, and increased frequency of occurrence of the most extreme events. This latter is not independent from the former, as in fact the maximal extreme event takes infinitely long time to occur again and because of an increase in $y_{\max }$ with driving, extremes of a magnitude that was maximal for the undriven case will have a finite frequency. To compare the relative frequency of extremal behavior for different time-scale factors $(\tau)$, regardless the $y_{\max }$ limit values that the behavior is bounded by, the kurtosis is commonly evaluated. We found that in the driven cases the kurtosis is also enhanced. The increased levels of $y_{\max }$ are consistent with the appearance of islands of larger extent in the $y$-direction, as exemplified by Fig. 1a vs. b, and depend nonmonotonically on $\tau$. (ii) As for moderately extreme events, the mean return time varies in a nonmonotonic manner with $\tau$, and can be larger or smaller relative to the undriven case. In the limit of increasingly fast driving the undriven problem is approached. This is attributed to a "resistance" of the attractor to fast driving.

Points (i) and (ii) are independent but both of them are related to the distribution of the values of $y$ realized over time. They are respectively reflected in (i) a longer and (ii) a broader or thinner tail. As for (ii), it is noted that the mean return times are related to the normalized cumulative distribution $F\left(y_{T}<y\right)$ by the Kac lemma, such that $\overline{t_{r}} \propto 1 / F\left(y_{T}<y\right)$ (Altmann and Kantz, 2005).

For comparison, results obtained with white noise driving (by replacing $\tilde{x}$ in (L84) by a random variable uniformly distributed in $[-17.5,17.5])$ are also shown in Fig. 7. Similarly to fast chaotic drivings, the graph deviates only slightly from that of the undriven case, but a slight increase in the frequency of extreme events applies uniformly over the considered range of thresholds.

To characterize the frequency of extreme events, we evaluate the distribution $P\left(t_{r}\right)$ of return times $t_{r}$ (Bunde et al., 2004) (inset of Fig. 7) with chaotic driving. The distribution appears to be consistent with an exponential decay, $\propto e^{-0.055 t_{r}}$. Such a form has been found in simple chaotic maps (Nicolis and Nicolis, 2009), and corresponds in general to approximately uncorrelated data (Blender et al., 2008), in agreement with the property of Poincare recurrences in chaotic systems (Altmann and Kantz, 2005). 


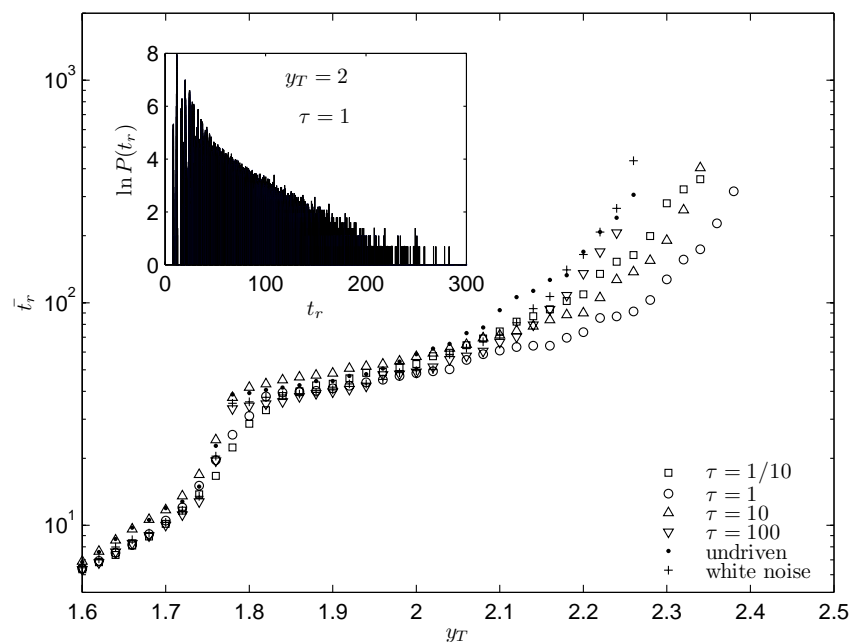

Fig. 7. Dependence of the mean return times $\bar{t}_{\mathrm{r}}$ on the threshold $y_{\mathrm{T}}$, based on the $y(t)$ time evolution over 500 time units. The undriven case $(\cdot)$ and the scenario of driving with white noise $(+)$ are included. Inset: distribution $P\left(t_{r}\right)$ of return times $t_{r}$ for $\tau=1$, based on data of about $4 \cdot 10^{5}$ values, represented on $2 \cdot 10^{4}$ bins.

\subsection{Extreme value statistics}

The extreme value statistics characterizes the tail of the distribution of model variable $y$. Therefore the maximal elements of large groups of consecutive maxima of the time series are considered. As an example, distributions of maximal elements in groups of 500 are displayed in Fig. 8 for $F(t)$ 's centered at different $F_{0}$ 's. The values of $F_{0}$ were chosen such that the excitation signal $F(t)$ sweeps regular $\left(F_{0}=6\right)$ or chaotic $\left(F_{0}=8\right)$ regimes of the bifurcation diagram (Fig. 1e), and also that it sweeps both regular and chaotic regimes $\left(F_{0}=7.5\right)$.

Distributions of such maxima in the asymptotic limit can be described by a three parameter $(E, \sigma, \gamma)$ family of generalized extreme value distributions (GEV), which combines the Gumbel $(\gamma \rightarrow 0)$, Fréchet $(\gamma>0)$ and Weibull $(\gamma<0)$ families (Coles, 2001). The functional form of the GEV distribution can be fitted to sample data in order to determine the prevailing type of distribution. The location $(E)$ and scale parameters $(\sigma)$ are the mean and standard deviation of the sample, and the shape parameter $\gamma$ is varied to minimize the fitting error. The fitting procedure is done iteratively by increasing the size of groups of consecutive maxima (Embrecht et al., 1997). For a fixed length of time series, the increasing group size will compromise the number of group maxima used for fitting, and therefore increasing errors in estimating $\gamma$ are incurred. The original time series length has to be long enough that an approximation of the limit value be possible with relatively small errors. For our analysis we generated a number of about $10^{7}$ maxima, with which an iterative estimation of the shape parameter resulted in the diagram shown in Fig. 9. From this an approximation of the limit values

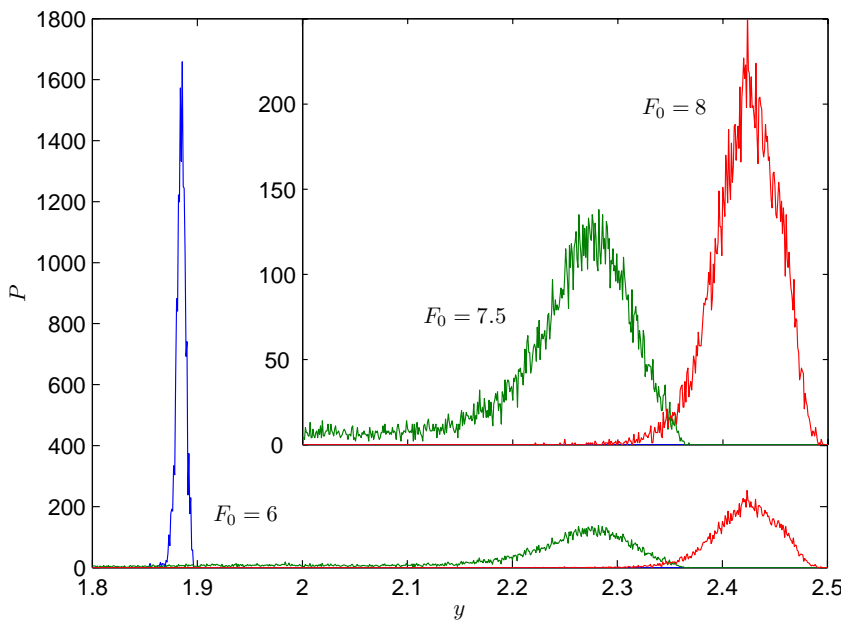

Fig. 8. Distribution (histogram) $P\left(y_{\mathrm{T}}\right)$ of maximal elements $y_{\mathrm{T}}$ in groups of 500 consecutive maxima of $y(t)$ for different values of $F_{0}$ (and $\tau=1$ ). Each histogram is constructed using 17000 data points, represented uniformly over 1000 bins in the range [1.6,2.5].

are obtained as $\gamma=-0.27,-0.38,-0.35$, respectively for $F_{0}=6,7.5,8$, which admit Weibull distributions in all cases. These findings, single-sided distributions, are in agreement with the fact that extreme events are limited by a finite maximum determined by the attractor of the driven L84 system. The shape parameter $\gamma$ is almost the same for the chaotic and mixed cases. In all cases $1 /|\gamma|>2$.

For a reference it is noted that uniform, triangular, and gaussian parent distributions generate exponential $(1 /|\gamma|=1)$, Rayleigh $(1 /|\gamma|=2)$, and Gumbel $(1 /|\gamma| \rightarrow \infty)$ extreme value distributions, respectively. With a continuous variation of $\gamma$, the case of the Rayleigh distribution constitutes a critical one when the tail of the distribution turns from convex to concave in a point of inflection. In all our cases the tails of the probability density of group maxima and also the (unknown) parent distribution are convex, i.e. they have gradients that are monotonically vanishing.

What is markedly different for the regular case, is the scale parameter $\sigma$, as indicated by Fig. 8. We have found the following values: $\sigma=0.0047,0.1191,0.0306$, respectively for $F_{0}=6,7.5,8$. These figures reveal another interesting effect that the greatest variability of extremes occurs for the mixed case.

\section{Discussions}

Our model climate is a fully deterministic one without any noise. The climate model L84 is a nonautonomous subsystem driven by the other subsystem L63. As a consequence of the driving, the method of snapshot (pullback) attractors can be applied. For the information dimension of the snapshot attractor the Kaplan-Yorke formula holds (Ledrappier 


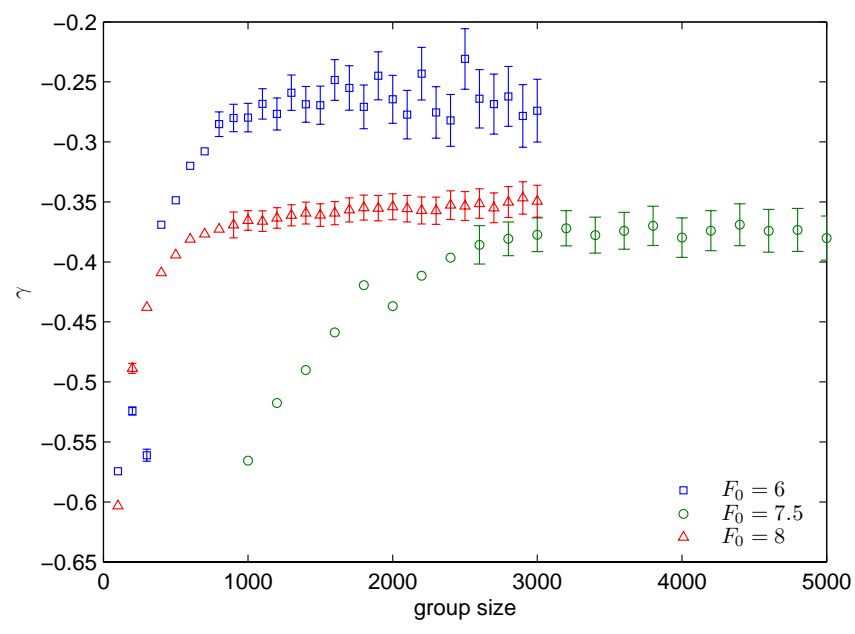

Fig. 9. Estimates of the shape parameter $\gamma$ of the GEV distribution for varying group size and for different values of $F_{0}$ (and $\tau=1$ ). The errorbars indicate the $95 \%$ confidence interval of estimation.

and Young, 1988) implying that the dimension is independent of time. The numerically determined values can, however, exhibit large fluctuations (Bódai et al., 2011).

We checked that other realizations of the driving (e.g. L63 initialized with a different state) do not modify the statistics. When the Rössler model (Tél and Gruiz, 2006, p. 190) is used for driving, the main characteristics (i) and (ii) remain the same. In comparison with this, when using L63 for driving, the convergence to the undriven problem is slower, which is believed to be due to the alternation between the "wings of the butterfly", whose time scale is longer than that of the motion on one wing. Approaching the undriven case entails the permanent presence of the island for sufficiently large $\tau$ 's. Having no "island" is thus an extreme event, not observed with fast driving. This is the case also for the white noise-driven dynamics, i.e. it has the characteristics of a fast driving.

As a deterministic driving has a nontrivial time scale, a resonance effect might occur and thereby enhance variability, i.e. the magnitude of extremes, and also their relative frequency. Indeed, we also evaluated the kurtosis of the distribution of variable $y$, and found a small but marked increase in resonance.

The structured chaotic "noise", with the possibility of varying time scales, thus, renders chaotic driving to be more interesting and realistic than white noise. This observation might lead to better predictions when applied to more realistic climate models. In a more general sense, in any kind of complex modeling where uncertainties are taken into account as noise, the use of chaotic driving might lead to increased variability than white noise.
Acknowledgements. Valuable comments by M. Ghil, Z. Rácz, H. Kantz, E. Altmann, N. Moloney, and I. M. Jánosi are gratefully acknowledged. This work was supported by OTKA no. NK72037, by the European Union and is co-financed by the European Social Fund (grant nos. TAMOP 4.2.1./B-09/KMR-2010-0002 and 0003).

Edited by: J. Kurths

Reviewed by: J. M. Redondo and another anonymous referee

\section{References}

Altmann, E. G. and Kantz, H.: Recurrence time analysis, longterm correlations, and extreme events, Phys. Rev. E, 71, 056106, doi:10.1103/PhysRevE.71.056106, 2005.

Arnold, L.: Random Dynamical Systems, Springer-Verlag, Berlin, 1998.

Blender, R., Fraedrich, K., and Sienz, F.: Extreme event return times in long-term memory processes near $1 / \mathrm{f}$, Nonlin. Processes Geophys., 15, 557-565, doi:10.5194/npg-15-557-2008, 2008.

Bódai, T., Károlyi, G., and Tél, T.: Fractal snapshot components in chaos induced by strong noise, Phys. Rev. E, 83, 046201, doi:10.1103/PhysRevE.83.046201, 2011.

Bunde, A., Eichner, J. F., Havlin, S., and Kantelhardt, J. W.: Return intervals of rare events in records with long-term persistence, Physica A, 342, 308-314, 2004.

Chekroun, M. D., Simonnet, E., and Ghil, M.: Stochastic climate dynamics: Random attractors and time-dependent invariant measures, http://adsabs.harvard.edu/abs/2010EGUGA. .1213469G, 2-7 May 2010.

Coles, S.: An Introduction to Statistical Modelling of Extreme Values, Springer, London, 2001.

Embrecht, P., Klüppelberg, C., and Mikosch, T.: Modeling Extremal Events for Insurance and Finance, Springer, 1997.

Freire, J. G., Bonatto, C., DaCamara, C. C., and Gallas, J. A. C.: Multistability, phase diagrams, and intransitivity in the Lorenz84 low-order atmospheric circulation model, Chaos, 18, 033121, doi:10.1063/1.2953589, 2008.

Ghil, M. and Jiang, N.: Recent forecast skill for the El Niño/Southern Oscillation, Geophys. Res. Lett., 25, 171-174, 1998.

Ghil, M., Chekroun, M. D., and Simonnet, E.: Climate dynamics and fluid mechanics: Natural variability and related uncertainties, Physica D, 327, 2111-2126, 2008.

Jacobs, J., Ott, E., Antonsen, T., and Yorke, J.: Modeling fractal entrainment sets of tracers advected by chaotic temporally irregular fluid flows using random maps, Physica D, 110, 1-17, 1997.

Ledrappier, F. and Young, L.-S.: Dimension formula for random transformations, Commun. Math. Phys., 117, 529-548, 1988.

Lorenz, E. N.: Deterministic Nonperiodic Flow, J. Atmos. Sci., 20, 130-141, 1963.

Lorenz, E. N.: Irregularity: a fundamental property of the atmosphere, Tellus A, 36A, 98-110, 1984.

Neufeld, Z. and Tél, T.: Advection in chaotically time-dependent open flows, Phys. Rev. E, 57, 2832-2842, 1998.

Nicolis, C. and Nicolis, G.: Memory effects in recurrent and extreme events, Phys. Rev. E, 80, 061119, doi:10.1103/PhysRevE.80.061119, 2009. 
Nicolis, C., Balakrishnan, V., and Nicolis, G.: Extreme Events in Deterministic Dynamical Systems, Phys. Rev. Lett., 97, 210602, doi:10.1103/PhysRevLett.97.210602, 2006.

Palmer, T. N.: Extended-Range Atmospheric Prediction and the Lorenz Model, B. Am. Meteorol. Soc., 74, 49-65, 1993.

Provenzale, A. and Balmford, N. J.: Chaos and Structures in Geophysics and Astrophysics, Woods Hole lecture notes, available from: http://www.whoi.edu/fileserver.do?id=21476\&pt=10\&p= 17353, 1999.

Roebber, P. J.: Climate variability in a low-order coupled atmosphere-ocean model, Tellus A, 47, 473-494, 1995.

Romeiras, F., Grebogi, C., and Ott, E.: Multifractal properties of snapshot attractors of random maps, Phys. Rev. A, 41, 784-799, 1990.
Shil'nikov, A., Nicolis, G., and Nicolis, C.: Bifurcation and predictability analysis of a low-order atmospheric circulation model, Int. J. Bif. Chaos, 5, 1701-1711, 1995.

Sommerer, J. C. and Ott, E.: Particles floating on a miving fluid: a dynamically comprehensive physical fractal, Science, 259, 335339, 1993.

Tél, T. and Gruiz, M.: Chaotic Dynamics, Cambridge University Press, New York, 190 pp., 2006.

Wilks, D. S.: Effects of stochastic parametrization on conceptual climate models, Philos. T. R. Soc. A., 366, 2475-2488, 2008. 